\title{
Pretraining a compound conditioned stimulus reduces unblocking
}

\author{
PETER C. HOLLAND \\ University of Pittsburgh, Pittsburgh, Pennsylvania
}

\begin{abstract}
Rats perceived pairings of a light + tone compound conditioned stimulus (CS) with either a highor low-value food unconditioned stimulus (US). Conditioning to the tone was blocked if the light had previously been paired with the same-value US. Considerable conditioning occurred to the tone if the light had been paired with the other-value US before compound conditioning. However, if after conditioning of the light, the compound was first paired with the same-value US, then subsequent pairings of the compound with the other-value US generated little conditioning to the tone. These results supported theories of blocking and unblocking that emphasize changes in the processing of the CS.
\end{abstract}

Kamin $(1968,1969)$ found that conditioning to an element $\mathrm{X}$ of a reinforced $\mathrm{AX}$ compound stimulus was blocked if the other element (A) had been previously paired with the reinforcer. Because conditioning to $\mathrm{X}$ depended on the reinforcement history of another stimulus, apart from X's relation to the reinforcer, this blocking phenomenon posed a major challenge to the contiguityor contingency-based conditioning theories of the time.

Two classes of theories emerged to account for blocking. One class ascribes blocking to conditioning-dependent changes in the effectiveness of the reinforcer, the unconditioned stimulus (US). For example, Kamin $(1968,1969)$ suggested that only surprising USs are effective reinforcers. Because the occurrence of the US is already predicted by the previously trained A, that US could not effectively reinforce learning to the added $\mathrm{X}$ element. Thus, $\mathrm{X}$ does not acquire conditioning because it is paired with an ineffective US.

The second kind of theory attributes blocking to changes in the effectiveness of the added conditioned stimulus (CS). For example, Mackintosh (1975) and Pearce and Hall (1980) argued that stimuli that do not provide new information about the occurrence of the US undergo decreases in attention or associability. Because the previous training of A allows it to predict the US, X rapidly loses its effectiveness as a CS. Thus, $\mathrm{X}$ does not acquire conditioning in a blocking experiment because it is not effectively processed together with the US.

Casually speaking, both of these types of theories claim that learning occurs only to the extent that the US is not predicted: If the organism is not surprised by the US, either that US is ineffective as a reinforcer, or the associability of the CS decreases rapidly so as to discourage further learning. Consistent with that claim is the observa-

This research was supported in part by a grant from the National Institute of Mental Health. Reprints are not available. Other correspondence can be sent to Peter Holland, Department of Psychology, University of Pittsburgh, Pittsburgh, PA 15260. tion of "unblocking": If the reinforcer is changed when $\mathrm{X}$ is introduced, substantial conditioning occurs to $\mathrm{X}$ (e.g., Dickinson, Hall, \& Mackintosh, 1976; Fowler, 1978; Holland, 1984; Rescorla \& Colwill, 1983). For example, Dickinson et al. (1976) found that considerable conditioning accrued to $X$ if $A$ was initially paired with a single shock and the AX compound was then paired with two shocks, or if A was initially paired with two shocks and $\mathrm{AX}$ was paired with a single shock.

However, Mackintosh and Turner (1971) presented data that favored "CS-effectiveness" accounts of blocking and unblocking. In one group of rats, A was first paired with a $0.5-\mathrm{mA}$ shock and $\mathrm{AX}$ was then paired with a $1.0-\mathrm{mA}$ shock. Unblocking, that is, conditioning to $\mathrm{X}$, was observed. In another group of rats, after pairings of $A$ with the $0.5-\mathrm{mA}$ shock, $\mathrm{AX}$ was also paired with the $0.5-\mathrm{mA}$ shock before it was paired with the $1.0-\mathrm{mA}$ shock. Unblocking did not occur in that group: $\mathrm{X}$ acquired little or no conditioned responding, despite its pairings with the 1.0-mA shock. That finding is anticipated within a theory like Mackintosh's (1975): Because the 0.5-mA shock was predicted by $\mathrm{A}$ when $\mathrm{X}$ was introduced, the associability of $\mathrm{X}$ decreased substantially. Consequently, when the intensity of the shock was increased, $X$ was ineffectively processed and hence could not acquire new learning. The absence of unblocking when $X$ is introduced before the reinforcer is changed is not consistent with a "US-effectiveness" account, however. According to such an account, although no conditioning should have accrued to $\mathrm{X}$ when it was introduced prior to the reinforcer shift, that shift should still have permitted additional conditioning to accrue to both $\mathrm{X}$ and $\mathrm{A}$.

The experiment reported here extended the data of Mackintosh and Turner (1971) in two ways. First, it examined the effects of introducing $X$ prior to shifting the value of the reinforcer on the unblocking observed both when the reinforcer value was shifted up and when it was shifted down. Although Mackintosh and Turner examined the effects of shifting from a $0.5-\mathrm{mA}$ shock to no shock, 
that procedure generated inhibitory, rather than excitatory, learning. Second, it used an appetitive conditioning procedure, which has several advantages for the study of blocking and unblocking phenomena. First, substantial unblocking effects have been observed with both upshifts and downshifts in reinforcer value in this preparation (Holland, 1984). Second, and perhaps more important, is the fact that the form of conditioned behavior exhibited in this preparation is substantially determined by the nature of the CS. For example, the visual CS used here evokes the behaviors rear and magazine, but the auditory CS used here evokes two different behaviors, startle, and head jerk (described below). Consequently, the source of conditioned responding to an auditory + visual compound CS (i.e., which element is responsible for the conditioned response) is determinable simply by examining the topography of the conditioned response; no element-alone probe trials are necessary. Furthermore, even if one element of the compound is already conditioned when the compound is introduced (as in a blocking procedure), trial-by-trial acquisition of conditioning to the added element can be detected as a topography shift.

Two groups of rats (Groups Up and Up-P) first received pairings of a visual CS with a low-value reinforcer $(\mathrm{L}+)$, and two groups (Groups Down and Down-P) received pairings of that visual CS with a high-value reinforcer $(\mathrm{L}++)$. Then Groups Up-P and Down-P received pairings of a compound of that stimulus and a novel auditory cue with the same reinforcer $(\mathrm{LT}+$ or $\mathrm{LT}++$, respectively). If prior training of $\mathrm{L}$ blocked conditioning to the added $\mathrm{T}$ cue, then responding characteristic of auditory CSs (head jerk and startle) would not emerge in this phase. Groups Up and Down remained in their home cages during this phase. Next, all groups received LT compound presentations reinforced with a shifted-value US. The value was shifted up in Groups Up and Up-P and down in Groups Down and Down-P. Again, head-jerk and startle behavior to the LT compound would emerge only to the extent that the added $\mathrm{T}$ element was associated with the US. Finally, conditioning to the T element alone was tested in all groups.

Previous data (Holland, 1984) suggests that substantial unblocking (i.e., conditioning to T) would occur in Groups Up and Down. The question of interest here is whether presentations of the compound prior to shifting the reinforcer's value would reduce the amount of unblocking, as found by Mackintosh and Turner (1971).

\section{METHOD}

\section{Subjects and Apparatus}

The subjects were 32 male Sprague-Dawley-strain rats 90-120 days old at the beginning of the experiment. All were experimentally naive. The rats were maintained at $80 \%$ of their ad-lib body weights throughout the experiment by limiting their access to food. They were housed individually in a colony that was illuminated between 6:00 a.m. and 8:00 p.m.

Eight experimental chambers, each $22.9 \times 20.3 \times 20.3 \mathrm{~cm}$, were used. The two end walls of each chamber were aluminum, and the side walls and top were clear acrylic. A dimly illuminated food cup was recessed in the center of one end wall. A 6-W jeweled panel light $6 \mathrm{~cm}$
Table 1

Experimental Procedure

\begin{tabular}{lccll}
\hline & Phase 1 & Phase 2 & Phase 3 & Test \\
\hline Group Up & L+ & & LT ++ & T- \\
Group Up-P & L + & LT + & LT ++ & T- \\
Group Down & L++ & & LT + & T- \\
Group Down-P & L++ & LT + & LT + & T- \\
\hline
\end{tabular}

Note-The rats in Groups Up and Down remained in their home cages during Phase 2. $L=$ houselight conditioned stimulus (CS); $T=$ tone $C S ; "+"=$ one pellet $U S ;$; $++"=$ one-then-two pellet US; “-" = no US.

above the food cup provided background illumination. The chamber floors were made of $.48-\mathrm{cm}$ stainless steel rods $1.9 \mathrm{~cm}$ apart. Each experimental chamber was enclosed in a sound-resistant shell that contained speakers for delivering auditory stimuli and a normally off 6-W houselight that served as one of the conditioned stimuli. The front wall of each shell contained an acrylic window to permit behavioral observations. Two low-light television cameras were mounted $2.1 \mathrm{~m}$ from the experimental chambers so that each could include four chambers in its field of view. Videocassette recorders were programmed to record behaviors occurring during, and $10 \mathrm{sec}$ before and after, CS presentations.

\section{Behavioral Observation Procedures}

All observations were made from videotapes. Each rat's behavior was observed at 1.25 -sec intervals during the 10 -sec period immediately prior to CS presentations and during the CS presentations. The observations were paced by auditory signals recorded on the videotapes. On each observation, one and only one behavior was recorded. Three behavioral categories were reported: magazine-standing motionless in front of the food magazine with head or nose within the recessed food cup; head jerk-short, rapid horizontal and/or vertical movements, usually (but not necessarily) directed toward the side of the chamber that contained the food cup; and startle - a rapid jump or change in position (see Holland, 1977, for more complete descriptions). Because the target tone stimulus did not evoke rear behavior, that behavior was not reported here.

\section{Procedure}

A summary of the procedure is found in Table 1. All rats were first trained to eat from the recessed food cup. Twelve deliveries of two 45$\mathrm{mg}$ food pellets were given on a variable-time 1-min schedule. After $20 \mathrm{~min}$, the rats were removed from the chambers.

Then, all rats received eight presentations of a 10-sec illumination of the houselight in each of eight 90 -min Phase 1 conditioning sessions. In Groups Up and Up-C, each light was followed with the delivery of a single 45-mg food pellet (low-value US). In Groups Down and Down-P, each light was followed with the delivery of one pellet followed $5 \mathrm{sec}$ later by the delivery of two more pellets (high-value US).

Next, the rats in Groups Up-P and Down-P received eight presentations of a 10-sec compound CS comprising the houselight and a 1,500$\mathrm{Hz}$ tone in each of four 90 -min Phase 2 sessions. Each compound CS was reinforced with the same reinforcer that was used in the houselight conditioning phase (i.e., the low-value US in Group Up-P and the highvalue US in Group Down-P). The rats in Groups Up and Down remained in their home cages during these sessions.

Then, all four groups received eight presentations of the light + tone compound in each of four 90 -min Phase 3 sessions. All groups received a shift in the value of the reinforcer during this phase: Each compound CS was reinforced with the high-value US in Groups Up and Up-P and with the low-value US in Groups Down and Down-P. Finally, all groups received a single 90 -min test session in which eight 10 -sec tone-alone CSs were presented, nonreinforced.

All statistical tests performed were two-tailed and used the $p<.05$ level of significance, unless otherwise noted.

\section{RESULTS}

In Phase 1, conditioning of the houselight proceeded more rapidly in the groups that received the high-value 
reinforcer than in those that received the low-value reinforcer. Over the final two sessions, magazine behavior was scored on means of $20 \%, 20 \%, 32 \%$, and $46 \%$ of all observations made during the houselight CS in Groups Up, Up-P, Down, and Down-P, respectively. The latter two groups combined showed reliably more magazine behavior than the former two groups $(U=57)$.

The left portion of each of the panels of Figure 1 shows responding to the LT compound during Phase 2 , in which Groups Up-P and Down-P received pairings of that compound with the reinforcer that was used in Phase 1 conditioning. Head-jerk behavior was acquired only to very low levels, and startle behavior showed a decrease in frequency (that behavior also occurs as an unconditioned or orienting response to novel auditory CSs-Holland, 1977). Thus, there was little evidence for substantial conditioning to the added tone when the reinforcer was not shifted. This outcome (blocking) is consistent with that observed by Holland (1984) with identical procedures.

The right portion of each of the panels of Figure 1 shows responding to the LT compound during Phase 3 , in which each group received pairings of $\mathrm{LT}$ with a shifted-value US. As Holland (1984) observed, substantial head-jerk and startle responding was acquired to the compound CS (presumably to the added tone element, which alone evokes those behaviors) in Groups Up and Down, which had had no previous LT training. However, intervening Phase 2 pairings of LT with the Phase 1 reinforcer substantially reduced the magnitude of those behaviors. Neither startle nor head-jerk behavior was acquired in Group Down-P, and Group Up-P showed no acquisition of startle behavior and only moderate levels of head-jerk behavior. Over all Phase 3 sessions, both head-jerk and startle behaviors were reliably more frequent in Group Up than in any of the other groups (Us $\leq 11)$ and were
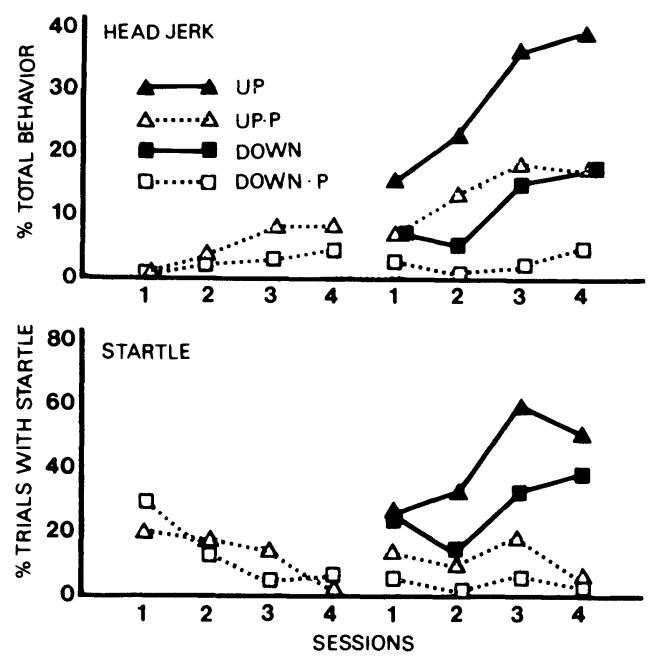

Figure 1. Responding to the houselightctone (LT) compound in Phases 1 and 2. Groups Up and Down remained in their home cages during Phase 2.

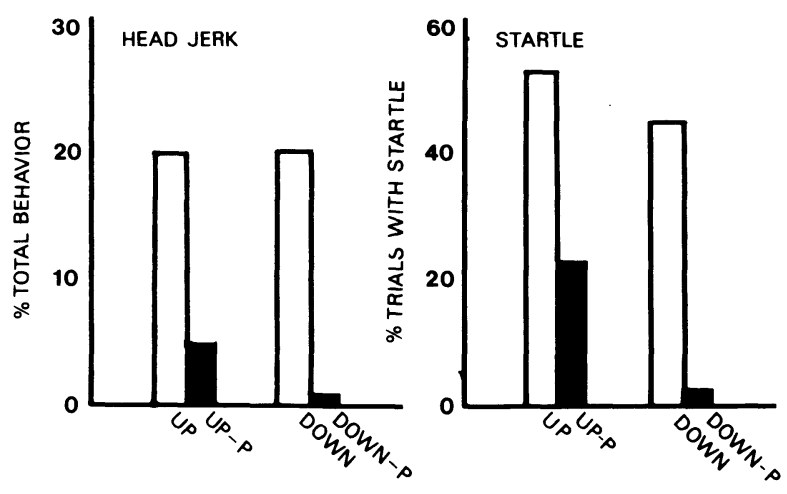

Figure 2. Responding to the tone $(\mathrm{T})$ in the test phase.

reliably greater in Group Down than in Group Down-P (Us $\leq 12.5$ ).

Figure 2 shows responding to the tone CS alone in the final test session. In general, the test data confirmed those of Phase 3. The left panel shows head-jerk behavior. Substantial head-jerk behavior occurred in the two groups that first received the compound CS when the reinforcer value was shifted (Groups Up and Down), but little or no headjerk behavior occurred in the two groups that received pairings of the compound with the Phase 1 reinforcer prior to the shift in US value (Groups Up-P and Down-P). Head-jerk behavior was significantly higher in each of the former two groups than in either of the latter two groups (Us $\leq 11.5)$. Groups Up and Down did not differ $(\mathrm{U}=$ 30), but Groups Up-P and Down-P differed marginally $(\mathrm{U}=17, \mathrm{p}<.12)$. The right panel of Figure 2 shows startle behavior. As with head-jerk behavior, substantial responding was observed in Groups Up and Down, and much lower levels of responding in Groups Up-P and Down-P. Startle behavior was reliably more frequent in Group Up than in either Group Up-P $(U=13.5)$ or Group Down-P $(U=1)$. Group Down showed significantly more startle behavior than did Group Down-P (U $=1$ ), but only marginally more than did Group Up-P (U $=15, \mathrm{p}=.08)$. Groups Up and Down did not differ $(\mathrm{U}$ $=25$ ), but Group Up-P showed reliably more startle behavior than did Group Down-P $(\mathrm{U}=11)$.

\section{DISCUSSION}

In Phase 2, there was little evidence for conditioning to $T$ in Group Up-P or Down-P when an LT compound was reinforced with the same reinforcer that was used in prior conditioning of $L$. However, when the same number of LT-US pairings were given in Groups Up and Down in Phase 3, in which the reinforcer's value was shifted either up or down (respectively), substantial conditioning to $T$ was acquired. Thus, both blocking and unblocking were observed with these conditioning parameters, replicating Holland's (1984) findings.

Most important, however, was the observation that pairings of the LT compound with the Phase 1 reinforcer prior to pairings of that compound with the shifted reinforcer substantially reduced the unblocking effect. That is, much less conditioning was observed to the added $\mathrm{T}$ element in Groups Up-P and Down-P than in Groups Up and Down, respectively, despite the former group's receiving the same number of 
pairings of $\mathrm{T}$ with the shifted US, plus additional pairings of $\mathrm{T}$ with the Phase 1 reinforcer.

These results are consistent with theories that attribute blocking and unblocking to changes in the effectiveness of the added CS. The preshift presentation of $\mathrm{T}$ in compound with a previously trained $\mathrm{L}$ would produce decrements in the associability of $\mathrm{T}$. Consequently, the associability of $\mathrm{T}$ at the beginning of compound training with the new, shifted reinforcer would be lower after such pretraining than if $\mathrm{T}$ were novel. Conversely, these data are inconsistent with theories that claim that blocking and unblocking effects are due solely to changes in the effectiveness of the US (e.g., Kamin, 1968, 1969). Those theories would not anticipate preshift training of LT to have substantial effects on conditioning to $T$ after a shift in the US value.

It is worth noting further than the most popular formalization of Kamin's notion, that of Rescorla and Wagner (1972), also fails to predict the occurrence of unblocking with downshifts in US value even without preshift exposure to the compound; that model instead anticipates the acquisition of conditioned inhibition to T (see Holland, 1984, Mackin tosh \& Turner, 1971, and Rescorla \& Colwill, 1983). However, Rescorla and Colwill (1983) noted that associations might be formed between the added $\mathrm{T}$ element and the pretrained $\mathrm{L}$ element in blocking and unblocking experiments. Consequently, responding to $\mathrm{T}$ might reflect not only its associations with the US, but also its associations with $\mathrm{L}$. The unblocking observed when the reinforcer value is shifted down may reflect T's association with $\mathrm{L}$ rather than with the US. Consistent with that claim, they reported that several manipulations thought to reduce the strength of responding due to such within-compound associations also reduced the magnitude of unblocking with downshifts in US value. But it is not clear how the preshift experience with the LT compound in the present experiment could be construed as reducing the strength of responding due to associations between $\mathrm{T}$ and $\mathrm{L}$; if anything, those $\mathrm{T}$ - $\mathrm{L}$ pairings might reasonably be anticipated to enhance $\mathrm{T}-\mathrm{L}$ association.

The greater test responding to $\mathrm{T}$ in Group Up-P than in Group Down-P suggests that the preshift compound training reduced unblocking produced by upshifts in reinforcer value more than it affected unblocking produced by downshifts. Two accounts for this finding come to mind. First, the greater responding in Group-Up may simply reflect generally higher levels of responding that are supported by the higher value reinforcer. This possibility is consistent with a theory like Mackintosh's (1975): As long as $\mathrm{T}$ is processed to some extent after preshift training of LT, more conditioning would be anticipated to $\mathrm{T}$ when it was paired with the higher value reinforcer, just as more conditioning to $L$ occurred with that reinforcer in Phase 1. Furthermore, for similar reasons, it would suggest that responding to the added element would be greater with the higher value reinforcer in blocking and unblocking procedures (Groups Up and Down) as well. Consistent with that claim, in Phase 3 Group Up showed more head-jerk behavior to LT (which presumably indexed unblocking, i.e., conditioning to the added tone) than did Group Down. But those differences were not evident in the test session, and Group Down did not show reliably less blocking than Group Up in Phase 2 (i.e., more head-jerk behavior to the LT compound).

Alternatively, the apparent difference in the magnitude of the LT pretraining effect may indicate that the mechanisms of unblocking in upshift and downshift procedures are not identical. For example, unblocking with downshift procedures may entirely involve CS-processing mechanisms, whereas unblocking with upshift procedures may also in- volve variations in US effectiveness. That claim is consistent with the present finding that preshift training of LT compound prevented unblocking with downshift procedures, but only reduced it with upshift procedures. It is also worth noting that Holland (1984) performed several manipulations that had greater effects on the responding that occurred with downshift procedures than on that observed with upshift procedures. However, it is not evident how any of those postconditioning manipulations (most of which involved changes in reinforcement of the conditioning context) could be construed as affecting CS-processing mechanisms. Nevertheless, it is likely to be fruitful to consider blocking, unblocking, and other related phenomena to be multiply determined. Future investigations should consider the circumstances under which the various mechanisms are influential.

\section{RERERENCES}

Dickinson, A., Hall, G., \& Mackintosh, N. J. (1976). Surprise and the attenuation of blocking. Journal of Experimental Psychology: Animal Behavior Processes, 2, 313-322.

FowlER, H. (1978). Cognitive associations as evident in the blocking effects of response-contingent CSs. In S. H. Hulse, H. Fowler, \& W. K. Honig (Eds.), Cognitive processes in animal behavior (pp. 109153). Hillsdale, NJ: Erlbaum.

Holland, P. C. (1977). Conditioned stimulus as a determinant of the form of the Pavlovian conditioned response. Journal of Experimental Psychology: Animal Behavior Processes, 3, 77-104.

Holland, P. C. (1984). Unblocking in Pavlovian appetitive conditioning. Journal of Experimental Psychology: Animal Behavior Processes, 10, $476-497$.

KAmin, L. J. (1968). Attention-like processes in classical conditioning. In M. R. Jones (Ed.), Miami Symposium on the Prediction of Behavior: Aversive Stimulation (pp. 9-32). Coral Gables, FL: University of Miami Press.

Kamin, L. J. (1969). Predictability, surprise, attention, and conditioning. In B. Campbell \& R. Church (Eds.), Punishment and aversive behavior (pp. 279-298). New York: Appleton-Century-Crofts.

MACKInTosh, N. J. (1975). A theory of attention: Variations in the associability of stimuli with reinforcement. Psychological Review, 82, 276-298.

MaCkintosh, N. J., \& TURNER, C. (1971). Blocking as a function of novelty of CS and predictability of UCS. Quarterly Journal of Experimental Psychology, 23, 359-366.

Pearce, J. M., \& Hall, G. (1980). A model for Pavlovian learning: Variations in the effectiveness of conditioned but not of unconditioned stimuli. Psychological Review, 106, 532-552.

Rescorla, R. A., \& Colwill, R. M. (1983). Within-compound associations in unblocking. Journal of Experimental Psychology: Animal Behavior Processes, 9, 390-400.

Rescorla, R. A., \& Wagner, A. R. (1972). A theory of Pavlovian conditioning: Variations in the effectiveness of reinforcement and nonreinforcement. In A. H. Black \& W. F. Prokasy (Eds.), Classical conditioning II (pp. 64-99). New York: Appleton-Century-Crofts.

(Manuscript received for publication January 7, 1985.) 